\title{
Low Friction Traction for Cervical Spine Dislocation
}

\author{
Lior Merom1,2, Michael Soudry1,2, Nahum Rosenberg1,2 \\ ${ }^{1}$ Spinal Surgery Unit, Orthopaedics Department, Rambam-Health Care Campus, Haifa, Israel \\ ${ }^{2}$ The Ruth and Bruce Rappaport Faculty of Medicine, Technion-Israel Institute of Technology, Haifa, Israel \\ Email: nahumrosenberg@hotmail.com
}

Received 6 July 2015; accepted 23 August 2015; published 26 August 2015

Copyright (C) 2015 by authors and Scientific Research Publishing Inc.

This work is licensed under the Creative Commons Attribution International License (CC BY). http://creativecommons.org/licenses/by/4.0/

(c) $\underset{\mathrm{EY}}{\mathrm{i}}$ Open Access

\begin{abstract}
In order to reduce the magnitude of the force applied to skull for treatment of acute cervical spine dislocation, we developed a method of skeletal traction based on reduction of friction forces under the patient's head. Traction force was applied to sculls of five patients with cervical fracture-dislocations. A difference in friction interface between the patient's head and shoulder girdle was created. The traction weight required for the reduction of the vertebral dislocation was significantly lower than an expected minimal traction weight in the commonly used techniques $(p=$ 0.013). The presented method permits an effective and safe reduction of dislocated cervical vertebra by a relatively low traction force.
\end{abstract}

\section{Keywords}

Fracture, Dislocation, Cervical Vertebra, Scull Traction

\section{Introduction}

Dislocation injuries to the cervical spine have a high probability of serious neurological sequela and therefore should be reduced promptly. Since the immediate recognition and treatment of this condition is crucial, an attempt of an emergency reduction is usually carried out. The most widely used method for emergency reduction of acute cervical spine dislocations is by application of gradual traction to the patients' skull [1]. By this method, tension forces are generated in the neck causing an adequate over-distraction between the dislocated facets of the dislocated and the adjacent vertebra with their eventual reduction [2]. In the resistant to this method cases, a relatively high distraction force, up to 30 - 35 kilograms of connected weights, might be required for a successful reduction in reasonable time period [3]. This high force is transmitted directly to the scull through the drilled holes in the temporal area and can cause local damage or avulsion. [4]. In order to reduce the magnitude of the 
traction force applied to skull, without interfering with the effectiveness of the traction procedure, we developed a method for partition of friction magnitude between the area under the patient head (low friction area) and patient's shoulders (high friction area), thus producing higher and therefore more effective traction force transmission to the cervical spine, which becomes an interface between these two areas (see Appendix). We hypothesize that by this method less traction force will be required for successful reduction of cervical vertebra dislocation.

\section{Materials and Methods}

\subsection{Study Group (Table 1)}

We used this technique in 5 consequent patients in the age range of 20 - 60 years, four men and one woman. These patients were injured following road accidents (three patients) and sports injuries (two patients). All the patients were fully conscious with no peripheral neurological deficits.

Two patients sustained dislocation of C2 vertebrae over C3, additional two patients had C7 dislocation over T1 and one patient with C5 over C6 dislocation .The pattern of the spinal dislocation in these patients is shown in the Table 1.

\subsection{Data Analysis}

The traction force that was required for a successful reduction of dislocation in this study group was compared to the elsewhere published data on the minimal traction force that is usually required for the successful reduction of cervical vertebra dislocations in patients who were treated by the conventional slow traction method [5]. For the comparison we used the paired $t$ test and $p<0.05$ was considered for a significant difference.

\subsection{Technique}

\subsubsection{Traction Application and Patient Positioning}

Patients were placed supine on the bed in a horizontal position with the head placed on the surface covered with a nylon (low friction surface) sheet (Figure 1). The shoulders lied on the usual cotton sheet (high friction surface). A tong traction device (Cone's caliper) [6] was inserted into the patient's skull in the temporal area, two centimeters above external auditory canal. A longitudinal traction was applied by connected weights. In this manner a neck becomes a transitional interface between the relatively fixed shoulders, which lie on high friction surface, and pulled by traction to head over a low friction surface.

\subsubsection{Traction Protocol}

The traction force was applied by additive 1 kilogram per hour, starting with 1 kilogram of initial traction. A radiographic monitoring of the cervical spine alignment was performed on one hour intervals by lateral cervical spine views with the consequent neurological follow up. An addition of 1 kilogram of weight to the traction was performed hourly until a radiographic evidence of successful reduction has been achieved. A muscle relaxant (Diazepam) was administered to reduce cervical muscle spasm in order to facilitate the reduction. The treatment

\begin{tabular}{|c|c|c|c|c|c|}
\hline $\begin{array}{l}\text { Patient number, } \\
\text { gender \& age }\end{array}$ & Injury following: & Dislocation of: & $\begin{array}{l}\text { Expected minimal } \\
\text { traction weight } \\
\text { (kilograms) [4] }\end{array}$ & $\begin{array}{l}\text { Low friction } \\
\text { traction weight } \\
\text { (kilograms) }\end{array}$ & $\begin{array}{l}\text { Time until } \\
\text { reduction } \\
\text { (hours) }\end{array}$ \\
\hline 1 man 35 years & Road accident & C2 - C3 & 2.7 & 1.0 & 1 \\
\hline 2 man 55 years & Sports accident & $\begin{array}{l}\mathrm{C} 7 \text { - T1 bilateral } \\
\text { facet dislocation }\end{array}$ & 8.1 & 4.0 & 4 \\
\hline 3 man 20 years & Road accident & $\begin{array}{l}\text { C5 - C6 unilateral } \\
\text { facet dislocation }\end{array}$ & 5.4 & 4.0 & 4 \\
\hline 4 woman 60 years & Sports accident & $\begin{array}{l}\text { C7 - T1 unilateral } \\
\text { facet dislocation }\end{array}$ & 8.1 & 4.0 & 4 \\
\hline 5 man 30 years & Road accident & C2 - C3 \& fracture of dens & 2.7 & 1.0 & 1 \\
\hline
\end{tabular}




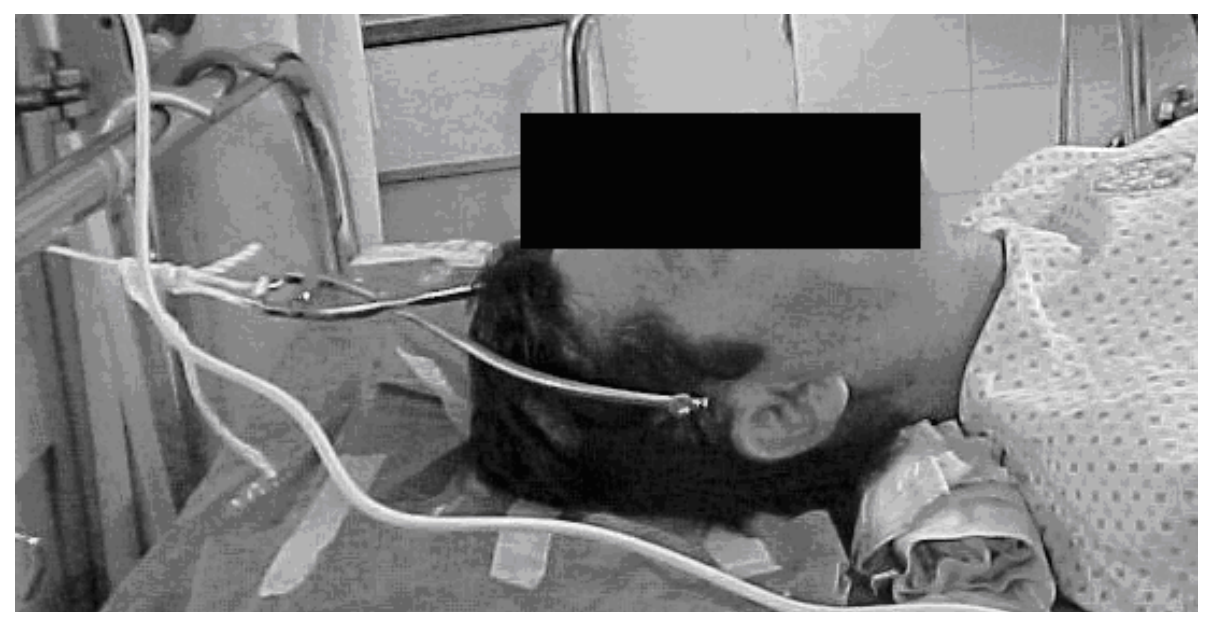

Figure 1. Patient treated by a slow traction over a low friction surface using Cone’s caliper.

team was instructed to stop the traction procedure if any evidence of neurological deficit appears, suggesting the neural canal compromise by a protruded intervertebral disk or bony spicule that might appear following this type of injury. Since no neurological deficits were detected before and during the traction procedure, the traction protocol was carried out without interruptions or a need for additional imaging procedures, e.g. CT scan or MRI scan, in all the studied patients.

\section{Results}

Traction weight that was required for the reduction of vertebral dislocation by this method was $37 \%-74 \%$ (mean 49\%) lower than expected by using the conventional slow traction technique $(p=0.013$ - paired $t$ test, Table 1). The mean time until successful reduction was 2.8 hours.

During the reduction procedure the patients were mechanically stable on their beds and there was no slippage of their heads from the supporting area covered by the low friction surface. No over distraction of the injured area in cervical spine was observed. No neurological deficits were observed following the treatment.

\section{Discussion}

Our initial results indicate that the described method of cervical spine dislocation reduction by traction over low friction surface being efficient, low cost and safe. With this modification, we showed a significant decrease of the required traction force without occurrence of pin site dislodgment or longitudinal slippage of the patient from the supporting surface. The authors were aware on a high degree of instability in the patients with bilateral facet dislocation and of the possibility of an acute neural canal obliteration by a protruded intervertebral disk or by bony fragments, therefore the patients were intensively followed, clinically and radiologically (by lateral radiographs of the cervical spine), in order to detect any appearance of neurological deficits or cervical over-distraction. None of those have been appeared in the present study group.

Since the method was applied on a small number of patients further large scale study will be required for its validation, but by this initial report, we intend to draw attention to the method's simplicity and effectiveness that should encourage its further effective implementation.

The presented technique permits an effective and safe reduction of cervical vertebra dislocation by lower than expected traction force applied to skull. This method should reduce the patients' discomfort and risk of pin dislodgment during treatment by traction.

\section{References}

[1] Durbin, F.C. (1957) Fractures-Dislocations of the Cervical Spine. Journal of Bone \& Joint Surgery, 39(B), 23-38.

[2] Miller, L.S., Cotler, H.B., Lucia, F,A., Cotler, J.M. and Hume, E.L. (1987) Biomechanical Analysis of Cervical Distraction. Spine, 12, 831-837. http://dx.doi.org/10.1097/00007632-198711000-00001 
[3] Norrel, H. (1978) Treatment of Unstable Spinal Fractures and Dislocations. Clinical Neurosurgery, 25, 193-208.

[4] Grundy, D.J. (1983) Skull Traction and Its Complications. Injury, 15, 173-177. http://dx.doi.org/10.1016/0020-1383(83)90008-6

[5] Leventhal, M.R. (2003) Fractures, Dislocations, and Fracture-Dislocations of Spine. In: Canale, S.T., Ed., Campbell’s Operative Orthopaedics. 10th Edition, Mosby, St Luis, London, Philadelphia, Sydney, Toronto, 1608.

[6] Cone, W. and Turner, W.G. (1937) The Treatment of Fracture-Dislocations of the Cervical Vertebrae by Skeletal Traction and Fusion. Journal of Bone \& Joint Surgery, 19(A), 584-602.

\section{Appendix}

If a mechanical system contains two masses, a trunk (m1) and a head (m2), then a longitudinal traction force applied to the head (m3xg) generates a tension force $(\mathrm{F})$ in the interface between the head and the trunk, i.e. in the neck (Figure A1). This force distracts the locked facets and causes reduction of the dislocated vertebra [5] and can be described as: $\mathrm{F}=\left(\mathrm{m} 3^{*} \mathrm{~g}-\mathrm{m} 2^{*} \mathrm{~g}^{*} \mu 2\right)-\mathrm{m} 1^{*} \mathrm{~g}^{*} \mu 1$ (g-gravitation coefficient, $\mu 1-$ friction coefficient of the surface under the trunk, $\mu 2$-friction coefficient of the surface under the head). If $\mathrm{m} 1, \mathrm{~m} 2, \mathrm{~m} 3$ and $\mu 1$ are kept constant then $\mathrm{F}=$ (constant $\mathrm{a})-($ constant $\mathrm{b}) * \mu 2$. Therefore a decrease in $\mu 2$ generates higher tension force (F) in the neck, without changing an applied traction mass (m3).

neck

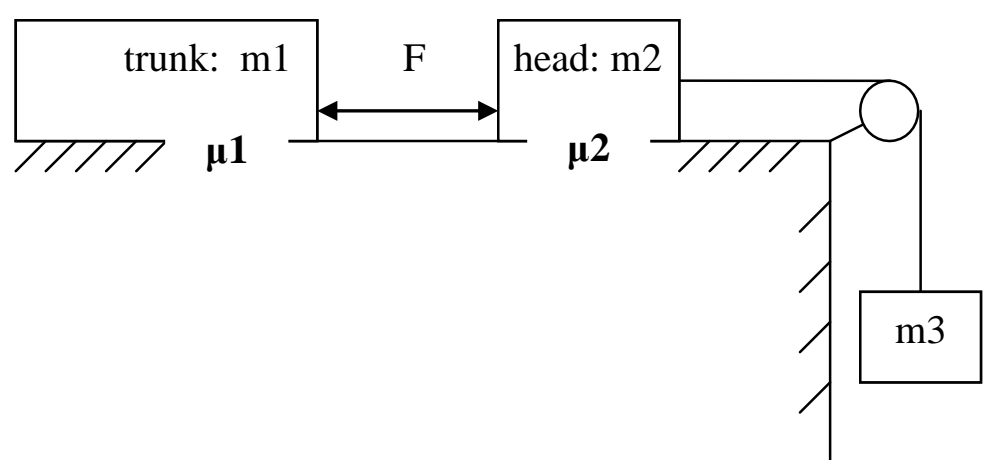

Figure A1. Schematic representation of the mechanical concept of the generated tension force (F) in the patient's neck following longitudinal scull traction. $\mathrm{m} 1$-mass of body trunk; $\mathrm{m} 2$-mass of head; m3 - mass of weights of an applied traction; $\mu 1$-friction coefficient of a surface under the trunk; $\mu 2$-friction coefficient of a surface under the head; F-tension force in the neck. 